Open Access

\title{
Active surveillance of prostate cancer: a questionnaire survey of urologists, clinical oncologists and urology nurse specialists across three cancer networks in the United Kingdom
}

Yiannis Philippou ${ }^{1}$, Hary Raja ${ }^{2}$ and Vincent J. Gnanapragasam ${ }^{2^{*}}$

\begin{abstract}
Background: Active surveillance is considered a mainstream strategy in the management of patients with low-risk prostate cancer. A mission-critical step in implementing a robust active surveillance program and plan its resource and service requirements, is to gauge its current practice across the United Kingdom. Furthermore it is imperative to determine the existing practices in the context of the recommendations suggested by the recent National Institute for Health and Clinical Excellence guidance on active surveillance of prostate cancer.

Methods: An internet questionnaire was circulated to urologists, clinical oncologists and urology nurse specialists across three geographically distinct cancer networks. Twenty five questions across four domains were assessed. (i) hospital resources (staff and clinical areas) utilised for active surveillance (ii) enrolment criteria (iii) follow up (iv) criteria that trigger conversion to active treatment.

Results: We received 35 responses, 20 of which were from urologists. The survey data suggests that there is marked heterogeneity in enrolment criteria with patients having features of intermediate-risk prostate cancer often recruited into Active Surveillance programs. Only $60 \%$ of our respondents use multiparametric MRI routinely to assess patient suitability for active surveillance. In addition, marked variation exists in how patients are followed up with regard to PSA testing intervals and timing of repeat biopsies. Only $40 \%$ undertake a repeat biopsy at 12 months. Tumour upgrading on repeat biopsy, an increase in tumour volume or percentage of core biopsies involved would prompt a recommendation for treatment amongst most survey respondents. In addition allocation of resources and services for active surveillance is poor. Currently there are no dedicated active surveillance clinics, which are well-structured, -resourced and -supported for regular patient counselling and follow up.

Conclusion: This variability in enrolment criteria and follow up is also demonstrated in international and national series of active surveillance. Resources are not currently in place across the UK to support an active surveillance program and a national discussion and debate to plan resources is much required so that it can become a mainstream therapeutic strategy.
\end{abstract}

Keywords: Prostate cancer, Active surveillance, Questionnaire survey

\footnotetext{
* Correspondence: vjg29@cam.ac.uk

${ }^{2}$ Academic Urology Group, Department of Surgery \& Oncology, University of Cambridge, Cambridge Biomedical Campus, Cambridge CB2 OQQ, UK Full list of author information is available at the end of the article
} 


\section{Background}

There is a clear trend, both in the UK and worldwide, towards managing patients diagnosed as having low-risk prostate cancer (LRPC) with active surveillance (AS) $[1,2]$. The recent publication of the Prostate Cancer Intervention Versus Observation Trial (PIVOT) has further added to the growing evidence which supports that LRPC can be safely managed by AS and without the treatment-related side effects [3]. There are however major caveats in considering widespread adoption of AS for LRPC in the UK and indeed in other health-care systems. Firstly, there is an increasing concern that the current diagnostic method of an elevated prostate specific antigen (PSA), digital rectal examination (DRE) and a single 10-12 core trans-rectal biopsy, carries a significant potential of missing higher risk disease. Secondly there remain no universally accepted inclusion criteria for AS and there is a lack of consensus on what an AS regime should consist of. Whereas there are national and international guidelines on standards of surgery and radiotherapy for prostate cancer these are lacking for AS [4]. Thirdly, the timing of PSA checks, repeat examination, place and role of imaging, repeat biopsies and triggers for intervention vary considerably from centre to centre and even from clinician to clinician. Finally, AS requires structured, well-resourced and supported clinics for regular patient reviews, and these will be needed for many years. It is therefore clear that an expansion of AS will be a significant resource implication for any health service let alone an already overstretched $\mathrm{Na}$ tional Health Service (NHS).

A move to a wider implementation of AS will require in addition to a uniform protocol, a national consensus on the resource and service requirements in setting this up and on the likely cost-implications of a long-term AS programme. As an initial step in this process the recent publication of the updated National Institute for Health and Clinical Excellence (NICE) guidance (CG175) has proposed a guideline for how men on AS may be managed (Table 1) [5]. A mission-critical step however in any attempt to adopt this guideline, is to gauge how AS is currently practiced in the UK.

\section{Methods}

We conducted an online survey of urologists, clinical oncologists (these were both medical and radiation oncologists with a special interest in uro-oncology) and urology nurse specialists with regard to the practice of AS within the East of England (EoE) cancer network. Data was collected during the year 2012-2013. The EoE cancer network delivers cancer care to 2.63 million people and is comprised mainly of two university hospitals and six district general hospitals. It employs approximately 50 consultant urologists. An internet questionnaire was circulated by email to urological departments of the
Table 1 Protocol for Active Surveillance as outlined by NICE: prostate cancer: diagnosis and treatment (CG175)

\begin{tabular}{|c|c|}
\hline Timing & Tests \\
\hline $\begin{array}{l}\text { At enrolment in active } \\
\text { surveillance }\end{array}$ & $\begin{array}{l}\text { Multiparametric MRI if not previously } \\
\text { performed }\end{array}$ \\
\hline \multirow[t]{4}{*}{ Year 1 of active surveillance } & Every 3-4 months: measure PSA \\
\hline & $\begin{array}{l}\text { Throughout active surveillance: } \\
\text { monitor PSA kinetics }\end{array}$ \\
\hline & Every 6-12 months: DRE \\
\hline & At 12 months prostate rebiopsy \\
\hline \multirow[t]{3}{*}{ Years $2-4$ of active surveillance } & Every 3-6 months: measure PSA \\
\hline & $\begin{array}{l}\text { Throughout active surveillance: } \\
\text { monitor PSA kinetics }\end{array}$ \\
\hline & Every 6-12 months: DRE \\
\hline \multirow{3}{*}{$\begin{array}{l}\text { Year } 5 \text { and every year thereafter } \\
\text { until active surveillance ends }\end{array}$} & Every 6 months: measure PSA \\
\hline & $\begin{array}{l}\text { Throughout active surveillance: } \\
\text { monitor PSA kinetics }\end{array}$ \\
\hline & Every 12 months: DRE \\
\hline
\end{tabular}

eight hospitals within the network. The email address of each urological department within each hospital was available on the hospital website. After receiving the email secretarial staff within each hospital were able to forward the questionnaire to all urological consultants, oncologists and urology nurse specialists working within each hospital. A further email was sent 1 month after in order to remind non-responders to complete the questionnaire. We also distributed the survey to two other cancer networks in geographically distinct areas of the UK. These were the North of England Cancer network and the Avon Somerset and Wiltshire cancer network. The reason behind the inclusion of a further two geographically distinct cancer networks was that we felt that this would allow us to form a more comprehensive opinion on the practice of AS across the UK and also allow comparison of our practice with other networks. Twenty five questions across four domains were assessed with associated multiple-choice answers (Additional file 1). Where more than one answer was possible respondents were able to select more options. The four domains were: (i) what hospital resources are currently utilised (staff and clinical areas) to counsel and follow up patients on AS (ii) enrolment criteria for AS (iii) how patients on AS are followed up (iv) respondents opinions on criteria that would trigger conversion to active treatment. Results were collated and rounded up to the closest percentage and represent the frequency of the answer selected for a particular question against the number of respondents answering the question.

\section{Ethics approval}

The above study is registered as an audit at Addenbrooke's hospital NHS trust. Ref: 3631. 
Table 2 Distribution of responses according to specialty

\begin{tabular}{lll}
\hline Specialty & Response percent (\%) & Response count \\
\hline Urology & 57 & 20 \\
Medical oncology & 0 & 0 \\
Clinical oncology & 20 & 7 \\
Urology specialist nurse & 20 & 7 \\
Oncology specialist nurse & 3 & 1 \\
Total & & 35 \\
Skipped question & & 0 \\
\hline
\end{tabular}

\section{Results}

Completed questionnaires were received from 15 urologists, six clinical oncologists and four specialist nurses within our cancer network with all invited trusts taking part. We received a further 10 responses from the other two cancer networks we surveyed (Table 2). From the 35 respondents, 31 were directly involved in managing patients on AS with most centres reported managing more than 30 men a year by AS.

\section{Current setting of AS}

Resources allocated to AS counselling were first assessed (Fig. 1). Dedicated urology prostate cancer clinics were used in $74 \%$ of cases but counselling and reviews also occurred in general urology clinics, oncology clinics, joint oncology and urology clinics as well as nurse led clinics. AS counselling was primarily done by urologists in $75 \%$ of cases. However oncologists and nurse specialists were also actively involved in this process. Respondents were then asked about the existence of an AS policy used in their unit. Of all respondents $68 \%$ reported the use of a policy to guide selection of men suitable for AS. The rest however stated of not being aware of any agreed policy. These results suggest that currently there is little evidence of a dedicated service for men managed by AS within current NHS resource provisions.

\section{Enrolment criteria for AS}

Respondents were then asked about different enrolment criteria used in enlisting patients to their AS programme (Fig. 2a). With regards to age, there was a strong agreement that AS would not be considered in men of 55 years or younger. A sizeable proportion (46\%) would not consider it in men over 75 years either with the preference here being for watchful waiting. There was a strong agreement also that a classical definition of low risk (Gleason score of 6, a PSA level of $\leq 10 \mathrm{ng} / \mathrm{ml}$ and a TNM stage of $\leq$ T2) were necessary for enrolment into an AS programme. Some would also consider a PSA level between 10 and $20 \mathrm{ng} / \mathrm{ml}$ as long as other characteristics such as Gleason score and TNM stage were favourable. $64.5 \%$ and $29 \%$ of respondents would consider patients for AS if the TNM stage was $\mathrm{T} 2 \mathrm{~b}$ or $\mathrm{T} 2 \mathrm{c}$ respectively. A significant majority considered the number of cores involved as well as the

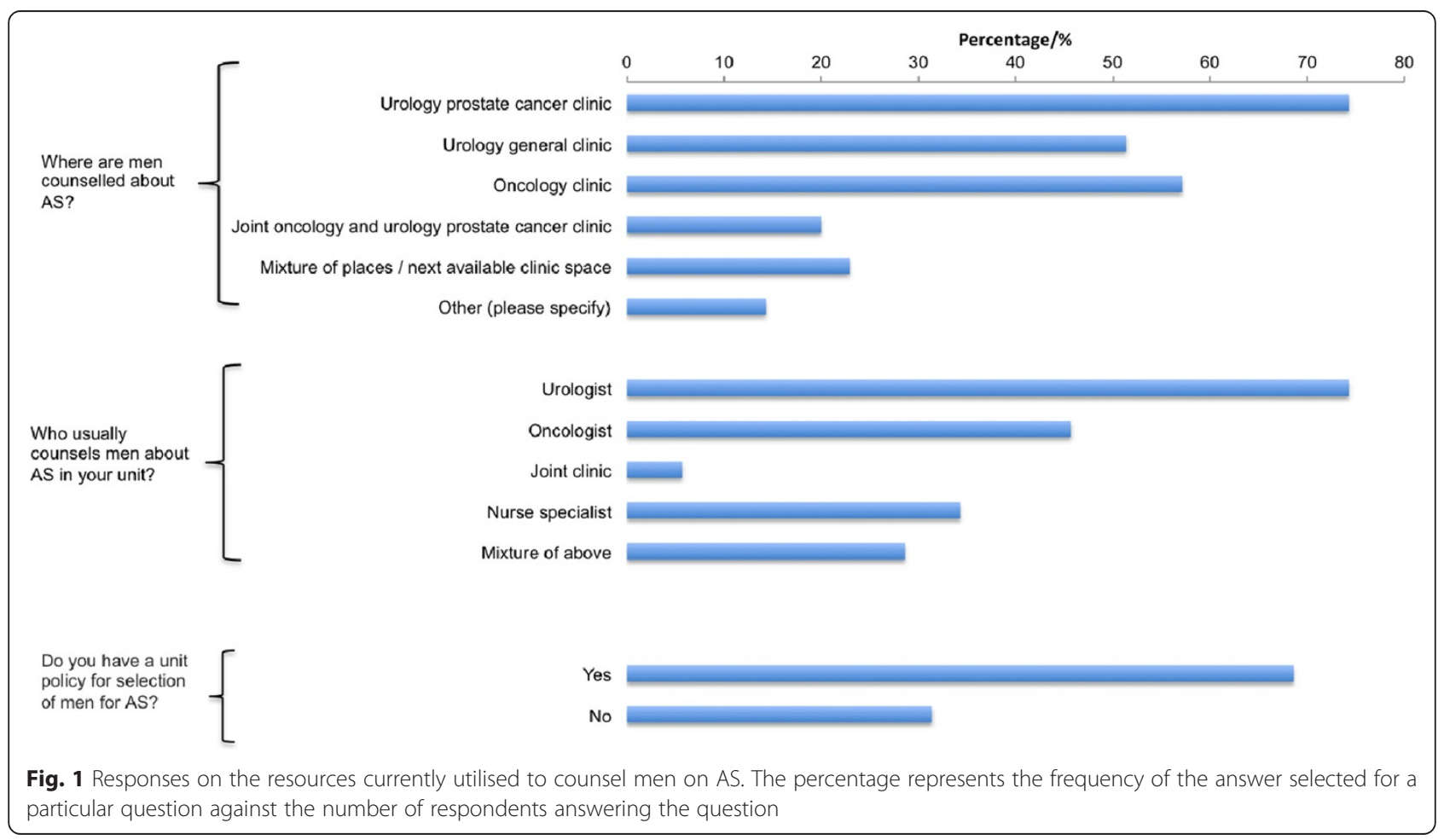




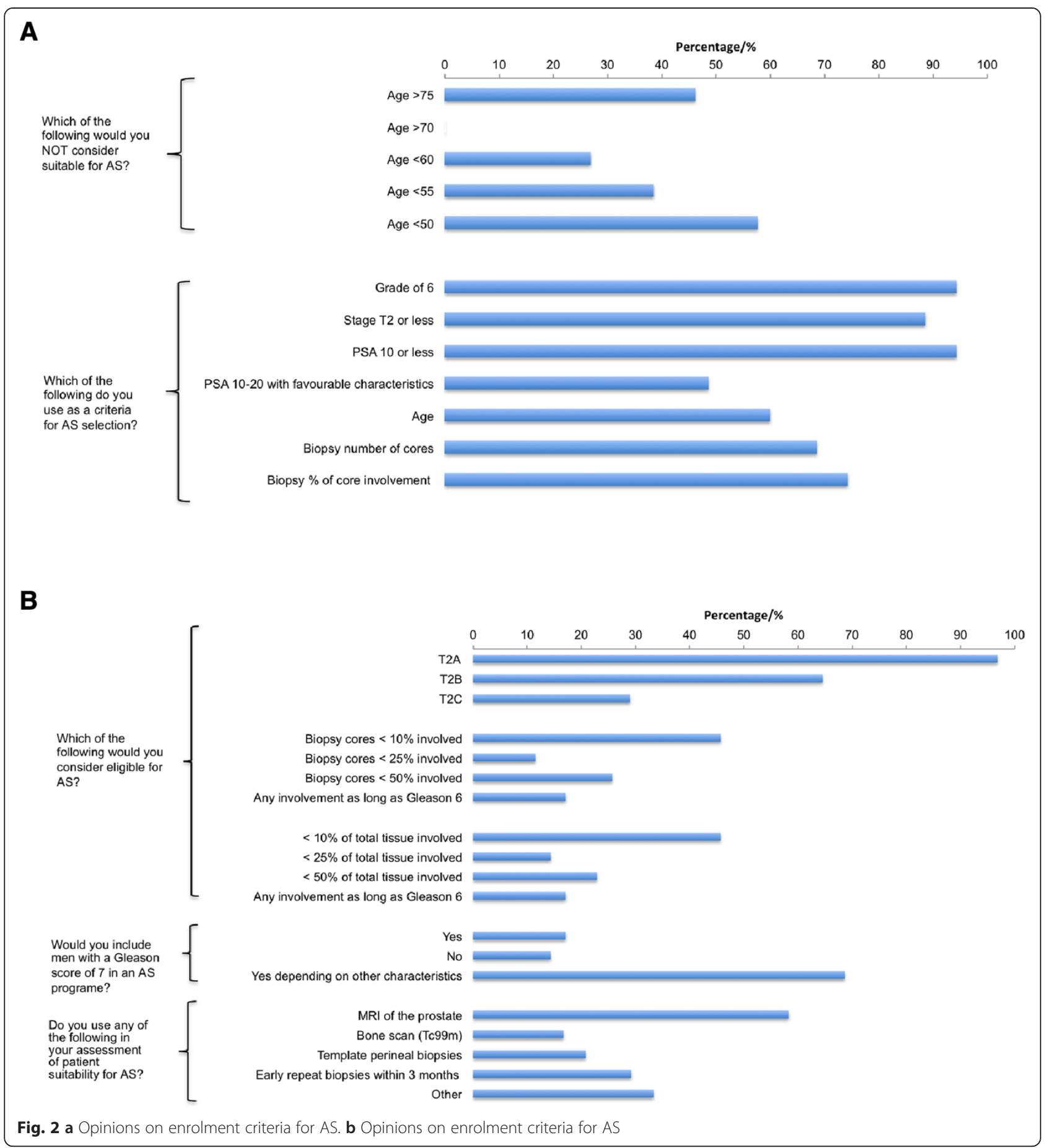

percentage of the core involved as important points in decision making for AS enrolment. Specifically, $17 \%$ would not be deterred from AS if more than $50 \%$ of total number of cores biopsied were involved as long as the Gleason score is 6 . We also observed that patients were being enrolled in programs with characteristics of intermediate risk prostate cancer (Fig. 2b). Interestingly, nearly $70 \%$ would consider AS in patients with a Gleason score of 7 if other tumour characteristics such as TNM stage, age, PSA level and information on biopsy core involvement were favourable. $58.3 \%$ routinely use multiparametric MRI (mp MRI) and $29.2 \%$ perform an early repeat biopsy (TRUS or template) within 3 months to aid in the selection of patients suitable for AS. These findings 
highlight the current significant variability and lack of standardisation in the inclusion criteria for men on AS across hospitals even within a single network.

\section{Follow up of patients on AS}

Respondents were asked about how they followed up patients on AS (Fig. 3a). The majority of respondents $(62 \%)$ indicated that they followed patients up with 3 monthly PSA at least in the first 2 years on AS and nearly $40 \%$ would carry out the first repeat biopsy within 12 months. A significant minority however would only rebiopsy if there was evidence of change in the serum PSA or other clinical changes. Most respondents (60\%) used DRE as an integral part of AS monitoring. The vast majority did not use MRI as a tool to monitor men on AS. Follow up was undertaken in a multidisciplinary setting with

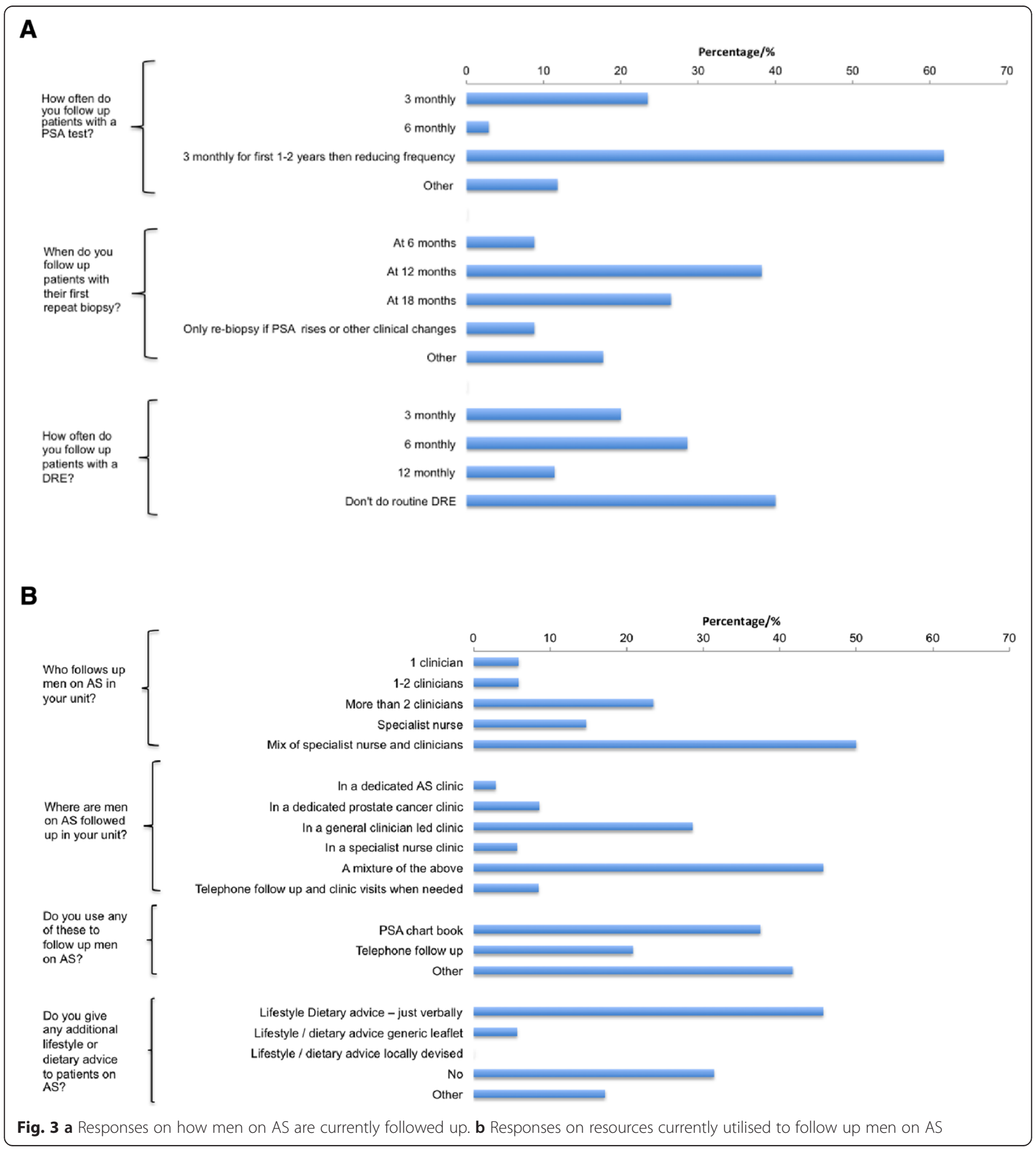


at least two clinicians or a mixture of clinicians and nurse specialists involved in patient care (Fig. 3b). This however did not often occur in dedicated AS prostate cancer clinics but mainly in clinician led general urology clinics. There was also a mixture of follow up methods including PSA chart books and telephone checks. A majority of respondents also stated that they felt it was important to give patients on AS certain life-style and dietary advice either verbally or in the form of a patient information leaflet. Finally, we asked what would trigger a recommendation for active treatment. Here there was broad agreement that tumour upgrading on repeat biopsy or an increase in tumour volume or percentage of core biopsies involved would prompt a recommendation for treatment. Half of our respondents however would recommend active treatment based on evidence of a rising PSA alone or an increase in PSA velocity. Finally changes in DRE findings as well as evidence of radiological progression of the tumour would prompt radical treatment in more than $50 \%$ of cases (Fig. 4).

\section{Comparison with other network trusts}

The same questionnaire was sent out to members of trusts part of two other UK cancer networks. We received a total of 10 responses, five from each cancer network. The findings were very comparable to our own network. There was broad agreement that men under 50 years would not be suitable for AS. In both networks surveyed, there was significant variation in respondent's views on inclusion criteria for AS (Fig. 5a). Similar to our data, most respondents would include Gleason grade 7 patients in an active surveillance programme but dependent on other clinical characteristics. In one of the two networks studied, a significant number of respondents advocated MRI and transperineal repeat biopsies as part of their assessment of patients suitability for AS. However only a minority of respondents in the other network used any additional biopsy or imaging in evaluating patients suitability. On the question of timing of repeat biopsies there was again variability in the responses (Fig. 5b). The majority advocated re-biopsy at 12 or 18 months after entry onto an AS programme. Finally, we compared triggers to initiate a change in management. Here there was broad consistency in terms of what would initiate a change and included an increase in grade and/or tumour volume. This comparison demonstrated that the lack of dedicated resources and variability in inclusion and follow up in AS is likely to be a universal issue across the NHS.

\section{Discussion}

In contemporary UK practice LRPC accounts for $20 \%$ of all new prostate cancer diagnoses [6]. The perception that LRPC is over-treated has gained the ascendancy among the urological community following results from randomised studies such as the European Randomised Study of Screening for Prostate Cancer (ERSPC) trial [7] and Prostate Cancer Intervention vs Observation Trial (PIVOT) [8]. It is therefore easy to foresee that AS will most likely become the preferred option of managing patients with LRPC. The UK NICE guidelines define men suitable for AS as having the following characteristics: clinical stage T1c; a Gleason score of $3+3$; a PSA density of < $0.15 \mathrm{ng} / \mathrm{mL} / \mathrm{mL}$; and cancer in $<50 \%$ of their total number of biopsy cores with $<10 \mathrm{~mm}$ of any core involved (http:// guidance.nice.org.uk/CG175) [5]. These recommendations are very similar to the European, American and Canadian urological guidelines which universally recommend that AS is suitable for patients with Gleason score of 6 or less $[1,2,9]$. Our survey demonstrated that the NICE guidelines regarding AS enrolment are generally followed. However, in certain cases patients are being recruited into AS programmes with characteristics of intermediate-risk

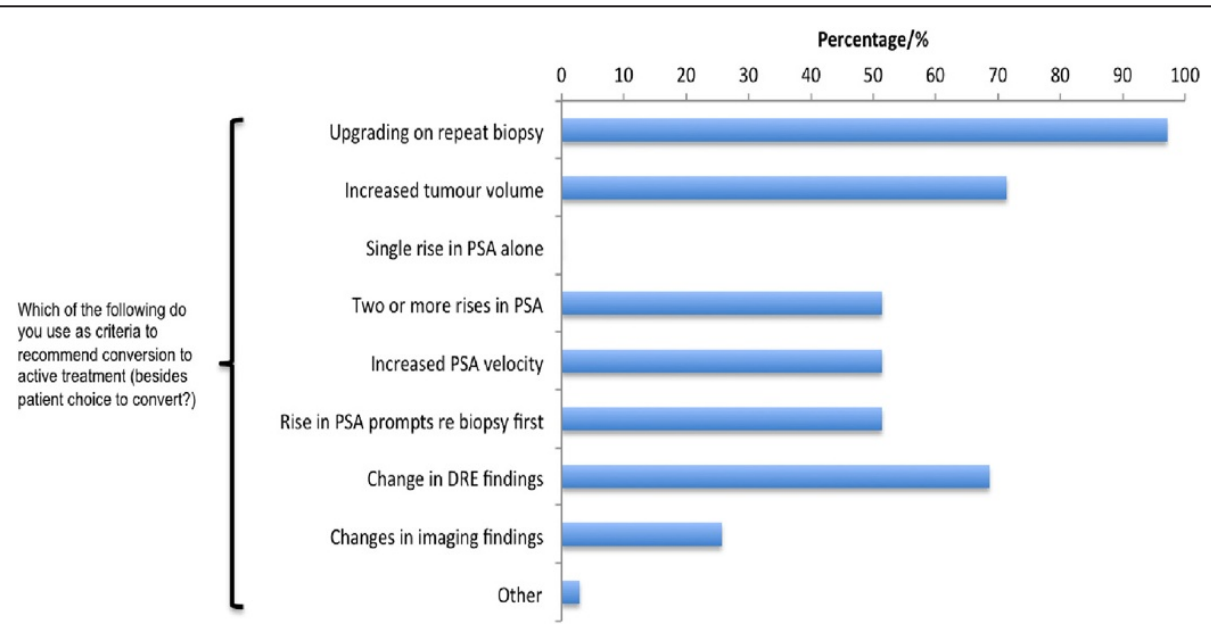

Fig. 4 Respondents views on criteria that would trigger conversion to active treatment 


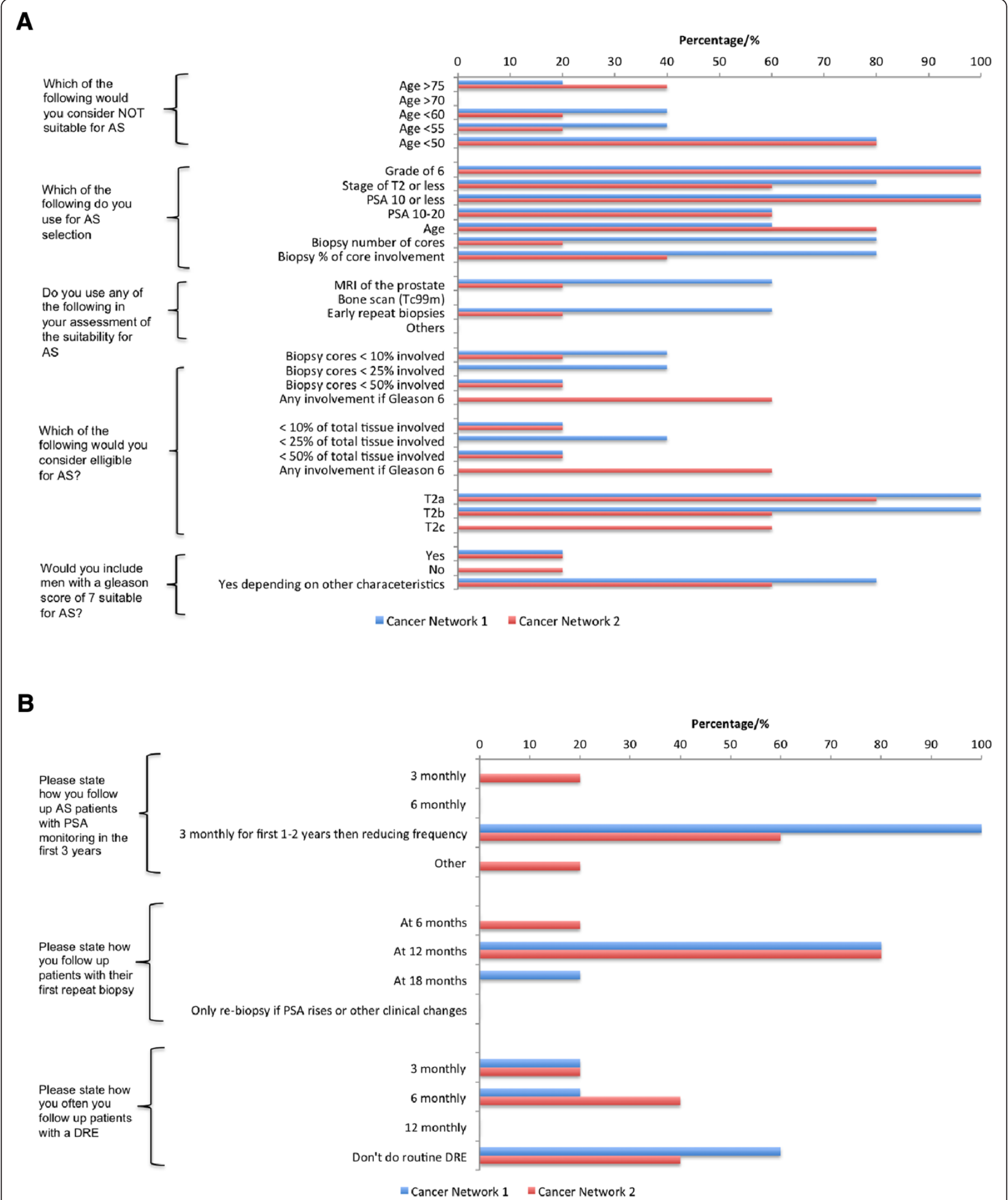

Fig. 5 a Respondents views on enrolment criteria for AS from the two geographically distinct cancer networks surveyed. b Respondents views on how they follow up patients on AS from the two geographically distinct cancer networks surveyed 
Table 3 Selection criteria for Active Surveillance in international published series

\begin{tabular}{|c|c|c|c|c|c|c|}
\hline Publication & Gleason Score & PSA (ng/ml) & Positive cores & $\%$ positive biopsy cores & $\begin{array}{l}\% \text { cancer involvement } \\
\text { per core }\end{array}$ & $\mathrm{CT}$ \\
\hline Dall' Era [1 1] & $\leq 6$ & $<10$ & - & $<33$ & - & $\leq 2 \mathrm{a}$ \\
\hline Bul [10] & $\leq 7$ & $<20$ & $\leq 3$ & - & - & - \\
\hline Soloway [12] & $\leq 6$ & $\leq 10$ & $\leq 2$ & - & $<20$ & $\leq 2$ \\
\hline Tosoian [18] & $\leq 6$ & $\leq 10$ & $\leq 2$ & - & $<50$ & $1 c$ \\
\hline Ercole [13] & $\leq 6$ & $<10$ & $\leq 2$ & - & $<50$ & $\leq 2 \mathrm{a}$ \\
\hline Klotz [14] & $\leq 6$ & $\leq 10$ & - & - & - & $\leq 2 \mathrm{~b}$ \\
\hline Ischia [15] & $\leq 6$ & $<10$ & - & - & - & $\leq 2 \mathrm{a}$ \\
\hline Thomsen [16] & $\leq 6$ & $\leq 10$ & $\leq 3$ & - & $<50$ & $\leq 2 \mathrm{a}$ \\
\hline Selvadurai [17] & $\leq 6$ & $<15$ & - & $\leq 50$ & - & $\leq 2$ \\
\hline
\end{tabular}

CT clinical tumour category, PSA prostate specific antigen

prostate cancer. A significant proportion of our respondents considered men for AS who had T2b or T2c disease, a PSA of $10-20 \mathrm{ng} / \mathrm{ml}$, a Gleason score of 7 and patients with tumour involvement of more than $50 \%$ of their total biopsy cores. This reflects that routine UK practice of AS commonly outstretches that described in most international published series of AS and also the recommendations of European, American and Canadian urological guidelines. Encouragingly however, there was a general agreement between our respondents as to the criteria that would trigger conversion to radical treatment.

Our survey demonstrated the marked heterogeneity which exists in the practice of AS across our cancer network. This variability is not unique to our cancer network alone but also evident in other cancer networks too in geographically distinct areas of the UK. This striking variability in AS enrolment criteria, follow up and triggers for intervention is also demonstrated in international and national series of AS (Tables 3 and 4). In these studies, patients were followed up with a combination of repeat biopsies, serial PSA measurements and clinical examination. The frequency of repeat biopsies varied widely analogous to our own cancer network. Some carried out biopsies annually [10-12], while others every 2 or 3 years [13-16], and in some depending on clinical characteristics $[17,18]$. Almost unanimously in the studies we reviewed tumour upgrading on repeat biopsy would prompt a recommendation for treatment. Some studies also considered an increase in tumour volume or percentage of core biopsies involved as a trigger to radical treatment. PSA doubling time or velocity was also sometimes a trigger to proceed to radical treatment.

The updated NICE guidance has advocated the use of mpMRI at the time of AS enrolment followed by a repeat biopsy at year one and has put in place a follow up regime as its key suggestions. To understand what the impact of these recommendations would be on routine clinical practice, our survey assessed the current reported patterns of practice. Amongst our respondents only $40 \%$ of respondents performed a repeat biopsy at 12 months and only $60 \%$ use mpMRI routinely as a tool for selecting patients suitable for AS. It should be noted however that although the NICE guidelines have clearly stated that mpMRI should occur at the time of AS enrolment, the EUA and AUA guidelines are not so prescriptive. Similarly Canadian urological guidelines from Ontario recommend that mPMRI may be included in AS protocols but is not currently a necessity $[1,2,9]$. In contrast, the recommendation on 3-4 monthly PSA checks is consistent with current routine practice in our survey. Even so there will need to be a method to monitor and track the PSA. Similarly recommendations for DRE every 6-12 months is

Table 4 Triggers to treatment used in patients under Active Surveillance

\begin{tabular}{|c|c|c|c|c|c|c|c|}
\hline Publication & $\begin{array}{l}\text { Gleason Score on } \\
\text { repeat biopsy }\end{array}$ & Positive cores & $\begin{array}{l}\% \text { cancer involvement } \\
\text { per single core }\end{array}$ & $\begin{array}{l}\% \text { positive } \\
\text { biopsy cores }\end{array}$ & PSAdt CT (years) & $\begin{array}{l}\text { PSAv } \\
\text { (ng/ml/year) }\end{array}$ & CT \\
\hline Dall'Era [11] & Progression & - & - & - & - & $>0.75$ & - \\
\hline Tosoian [18] & $>6$ & $>2$ & $>50$ & - & - & - & - \\
\hline Ercole [13] & Progression & Increase & Increase & - & - & - & Upstage \\
\hline Klotz [14] & $\geq 4$ & - & - & - & 3 & - & - \\
\hline Ischia [15] & Progression & - & - & - & - & - & Upstage \\
\hline Thomsen [16] & $\geq 3+4$ & $>3$ & - & - & 3 & - & Upstage \\
\hline Selvadurai [17] & $\geq 4+3$ & - & - & $>50$ & - & $>1$ & - \\
\hline
\end{tabular}

cT clinical tumour category, PSAdt prostate-specific antigen doubling time, PSAv PSA velocity 
probably best combined with clinic reviews. Of note, in our survey there was great variability in who did the AS follow up including DRE. Moreover $40 \%$ of respondents did not routinely perform a DRE during the follow up appointment. Again adherence to the NICE guidelines will require a significant change in practice and ideally necessitate consistency in AS follow up providers.

From the results of this survey it is quite clear that the implementation of a robust and consistent AS program according to the recommendations of the NICE guidelines will prove challenging due to the current variation in its practice. In addition the burden of follow-up with clinical examinations and serum PSA testing on both men and healthcare systems is far from cost-neutral. Even more so the use of novel strategies such as mpMRI at the time of AS enrollment will further put a strain on radiology providers. It is clear that in addition to reducing the variability in the practice of AS there is also the need for robust cost-effectiveness studies to ensure that such novel strategies are both clinically and cost-effective.

This study however does not come without its limitations. Firstly the methodology of this study is a questionnaire survey study with a total of 35 respondents which is a low number of participants and may not accurately depict the practice of AS within the EoE cancer network. Also this study was UK specific and may not mirror the trends in the world-wide practice of AS though our review of other international published series of AS did demonstrate variability in its practice. Furthermore the majority of our respondents were from primarily academic institutions therefore it is likely that the practice of AS is even more heterogeneous outside the academic setting.

\section{Conclusions}

Despite its limitations, the present survey has demonstrated the marked heterogeneity in which AS is practiced across a cancer network in the UK. This is also mirrored across other hospitals in the UK. It is clear that if the NICE guidelines are adopted then the resource requirements are likely to be very significant and not currently in place across the UK. These issues need urgent resolution and now is the time for a national discussion and planning. This would provide the much needed reassurance to clinicians and patients of the robustness of an AS programme on a par with current standard therapeutic options.

\section{Additional file}

Additional file 1: Questions included in survey.

\section{Abbreviations}

LRPC: Low-risk prostate cancer; AS: Active Surveillance; EoE: East of England; PIVOT: Prostate Cancer Intervention Versus Observation Trial; PSA: Prostate specific antigen; DRE: Digital Rectal Examination; NICE: National Institute for Health and Clinical Excellence; NHS: National Health Service; mp MRI: Multiparametric MRI.

\section{Competing interest}

The authors declare that they have no competing interests.

\section{Authors' contributions}

YP contributed to the data analysis and manuscript writing. HR contributed to data collection and analysis. VJG conceived the project and contributed to data collection, data analysis and final manuscript approval. All authors read and approved the final manuscript.

\section{Author details}

${ }^{1}$ Department of Surgery, Basildon \& Thurrock University Hospital, Essex SS16 5NL, UK. ${ }^{2}$ Academic Urology Group, Department of Surgery \& Oncology, University of Cambridge, Cambridge Biomedical Campus, Cambridge CB2 OQQ, UK.

Received: 4 January 2015 Accepted: 3 June 2015

Published online: 13 June 2015

\section{References}

1. Thompson I, Trasher JB, Aus G, Burnett AL, Canby-Hagino ED, Cookson MS, et al. AUA Prostate Cancer Clinical Guideline Update Panel. Guideline for the management of clinically localised prostate cancer: 2007 update. J Urol. 2007;177:2106-31.

2. Heidenreich A, Bellmunt J, Bolla M, Joniau S, Mason M, Matveev V, et al. EUA guidelines on prostate cancer. Part 1: screening, diagnosis and treatment of clinically localised disease. Eur Urol. 2011;59:61-71.

3. Wilt TJ, Brawer MK, Jones KM, Barry MJ, Aronson WJ, Fox S, et al. Radical prostatectomy versus observation for localized prostate cancer. N Engl J Med. 2012;367:203-13.

4. Dall'Era MA, Albertsen PC, Bangma C, Caroll PR, Carter HB, Cooperberg MR, et al. Active surveillance for prostate cancer: a systematic review of the literature. Eur Urol. 2012;62:976-83.

5. NICE guideline on prostate cancer: diagnosis and treatment. February 2014 (CG175). Available at http://www.nice.org.uk/guidance/cg175. Accessed October 2014

6. National Institute for Health and Clinical Excellence. Costing statement: prostate cancer: diagnosis and treatment. Implementing the NICE guidance on prostate treatment(CG175). Available at: https://www.nice.org.uk/ guidance/cg175/resources/cg175-prostate-cancer-costing-statement2. Accessed August 2014

7. Schröder FH, Hugosson J, Roobol MJ, Tammela TL, Ciatto S, Nelen V, et al. Screening and prostate-cancer mortality in a randomized European study. N Engl J Med. 2009;360:1320-8.

8. Bill-Axelson A, Holmberg L, Ruutu M, Garmo H, Stark JR, Busch C, et al. Radical prostatectomy versus watchful waiting in early prostate cancer. N Engl J Med. 2011;364:1708-17.

9. Cancer Care Ontario. Active surveillance for the management of localized prostate cancer. Available at www.cancercare.on.ca. Accessed March 2015

10. Bul M, van den Bergh RC, Zhu X, Rannikko A, Vasarainen H, Bangma CH, et al. Outcomes of initially expectantly managed patients with low or intermediate risk screen-detected localized prostate cancer. BJU Int. 2012;110:1672-7

11. Dall'Era MA, Konety BR, Cowan JE, Shinohara K, Stauf F, Cooperberg MR, et al. Active surveillance for the management of prostate cancer in a contemporary cohort. Cancer. 2008;112:2664-70.

12. Soloway MS, Soloway CT, Eldefrawy A, Soloway MS, Soloway CT, Eldefrawy $A$, et al. Careful selection and close monitoring of low-risk prostate cancer patients on active surveillance minimizes the need for treatment. Eur Urol. 2010;58:831-5.

13. Ercole B, Marietti SR, Fine J, Albertsen PC. Outcomes following active surveillance of men with localized prostate cancer diagnosed in the prostate specific antigen era. J Urol. 2008;180:1336-9.

14. Klotz L, Zhang L, Lam A, Nam R, Mamedov A, Loblaw A. Clinical results of long-term follow-up of a large, active surveillance cohort with localized prostate cancer. J Clin Oncol. 2010;28:126-31. 
15. Ischia JJ, Pang CY, Tay YK, Suen CF, Aw HC, Frydenberg M. Active surveillance for prostate cancer: an Australian experience. BJU Int 2012;109:40-3.

16. Thomsen FB, Roder MA, Hvarness H, Iversen P, Brasso K. Active surveillance can reduce overtreatment in patients with low-risk prostate cancer. Dan Med J. 2013;60:A4575.

17. Selvadurai ED, Singhera M, Thomas K, Mohammed K, Woode-Amissah R, Horwich A, et al. Medium-term outcomes of active surveillance for localised prostate cancer. Eur Urol. 2013;64:981-7.

18. Tosoian JJ, Trock BJ, Landis P, Feng Z, Epstein Jl, Partin AW, et al. Active surveillance program for prostate cancer: an update of the Johns Hopkins experience. J Clin Oncol. 2011;29:2185-90.

\section{Submit your next manuscript to BioMed Central and take full advantage of:}

- Convenient online submission

- Thorough peer review

- No space constraints or color figure charges

- Immediate publication on acceptance

- Inclusion in PubMed, CAS, Scopus and Google Scholar

- Research which is freely available for redistribution 